\title{
A Note on the Use-Classification of Four Digit Industries or
}

\section{How to Call a Spade a Spade}

by

\section{Gordon C. Winston \& ARThur MacEwan*}

Much of our understanding of structural change with industrialization is based on empirical studies that describe patterns in the relationship between consumption, intermediate and capital goods $[1 ; 2$, for instance]. Thus, classification of an industry's output, prices, imports or exports by use is of primary importance.

Even if the data available describe individual products, there is some ambiguity in use classification-safety razor blades (metal products) are clearly consumption goods but sewing machines (nonelectric machinery) are consumption goods if owned by housewives and capital goods if owned by tailors. Far more serious problems, however, arise when large industry sectors, like whole four digit industries, must be classified by use. If there is no basis for dividing any industry's output (or prices or imports or exports) among its alternative uses, then the entire industry must be classified as a single lump in one of the three use categories on the basis of some judgment - even if heroic - or else such aggregation by use must be abandoned.

But ad hoc classifications that lump entire four-digit industries under one or another heading are not appropriate if there exists a reasonable basis for dividing each industry's output among uses. Then, instead of assigning all metal products to capital goods (as did Chenery) it is possible to assign some portion to consumption goods (representing razor blades, et al), some to intermediate goods (rivets) and some to capital goods (spades).

To represent a step in the right direction, any system of division need only improve on the whole-industry classification. Such a system obviously can be based on the allocations of output shown in a country input-output table if changes over the period of the data in an industry's allocation are deemed to introduce a less serious error than that of whole-industry classification.

* The authors are Research Adviser and Research Associate respectively at the Pakistan Institute of Development Economics. They gratefully acknowledge the computational assistance renderad by Mr. A. D. Bhatti, also of the Institute. 
To illustrate this sort of industry classification and to show what it means in a study of structural change, we have used industry allocation from the TimsStern Table for Pakistan (1963/64) [3] to recompute two columns of Lewis and Soligo's study of Pakistan's industrial structure-the columns that show growth of gross output by four-digit industry between 1954/55 (averaged) and 1963/64 (fiscal) [2, Table A-4, p. 126]. Against Lewis and Soligo's growth rates, Table I reveals a lower rate for intermediate goods and a higher rate for consumption goods.

While Table I is self-explanatory, it is worth noting the contradictions in classification in cases like wood and furniture or chemicals and pharmaceuticals. Of course, one can argue with the input-output assignments of an industry's output (the high consumption of jute textiles) even while accepting this as a better approximation of what the use-categories are intended to describe.

\section{TABLE I}

\section{A WEIGHTED USE-CLASSIFICATION OF INDUSTRY OUTPUT}

\begin{tabular}{|c|c|c|c|c|c|c|}
\hline \multirow{3}{*}{$\begin{array}{l}\text { Industry } \\
\text { (1) }\end{array}$} & \multicolumn{3}{|c|}{ Lewis and Soligo } & \multicolumn{3}{|c|}{ Our division } \\
\hline & \multirow{2}{*}{$\begin{array}{c}\text { Weight* } \\
(2)\end{array}$} & \multicolumn{2}{|c|}{ Output at factor cost } & \multirow{2}{*}{$\begin{array}{c}\text { Weight* } \\
(5)\end{array}$} & \multicolumn{2}{|c|}{ Output at factor cost } \\
\hline & & $\begin{array}{l}1954 / 55 \\
\text { (3) }\end{array}$ & $\begin{array}{l}1963 / 64 \\
(4)\end{array}$ & & $\begin{array}{l}1954 / 55 \\
(6)\end{array}$ & $\begin{array}{c}1963 / 64 \\
(7)\end{array}$ \\
\hline \multicolumn{7}{|l|}{ A. Consumption Goods } \\
\hline Sugar & 1 & 97,576 & 383,835 & 1.000 & 97,576 & 383,835 \\
\hline Edible oịls & 1 & 92,175 & 574,294 & 0.813 & 74,938 & 466,901 \\
\hline Tea & 1 & 132,415 & 183,351 & 1.000 & 132,415 & 183,351 \\
\hline Food & 1 & 19,447 & 64,260 & 1.000 & 19,447 & 64,260 \\
\hline Beverages & 1 & 17,938 & 26,055 & 1.000 & 17,938 & 26,055 \\
\hline Tobacco & 1 & 72,639 & 404,422 & 1.000 & 72,639 & 404,422 \\
\hline Cotton \& other textiles & is 1 & 631,721 & $2,010,320$ & 0.873 & 551,492 & $1,755,009$ \\
\hline Jute textile & 0. & - & - & 0.768 & 64,297 & 397,727 \\
\hline Silk \& Artsilk & 1 & 35,256 & 105,509 & 1.000 & 35,256 & 105,509 \\
\hline Footwear & 1 & 40,200 & 84,220 & 1.000 & 40,200 & 84,220 \\
\hline Wood and furniture & 1 & 3,291 & 20,010 & 0.278 & 915 & 5,563 \\
\hline Paper & 0 & - & - & 0.087 & 2,654 & 13,810 \\
\hline Printing and publishing & g 1 & 37,164 & 119,160 & 1.000 & 37,164 & 119,160 \\
\hline Leather & 0 & - & - & 0.850 & 31,468 & 161,441 \\
\hline Rubber and other goods & ds 0 & - & - & 0.745 & 9,888 & 31.487 \\
\hline Soaps & 1 & 23,750 & 110,671 & 1.000 & 23,750 & 110,671 \\
\hline Matches & 1 & 27,683 & 47,490 & 1.000 & 27,683 & 47,490 \\
\hline $\begin{array}{l}\text { Chemicals \& phar- } \\
\text { maceuticals }\end{array}$ & $\mathbf{0}$ & - & - & 0.720 & 26,122 & 188,402 \\
\hline Petroleum & 0 & - & - & 0.830 & 59,719 & 185,098 \\
\hline
\end{tabular}


TABLE I (Contd.)

A WEIGHTED USE-CLASSIFICATION OF INDUSTRY OUTPUT

\begin{tabular}{|c|c|c|c|c|c|c|c|}
\hline \multirow{3}{*}{$\begin{array}{l}\text { Industry } \\
\text { (1) }\end{array}$} & \multicolumn{4}{|c|}{ Lewis and Soligo } & \multicolumn{3}{|c|}{ Our division } \\
\hline & \multirow{2}{*}{\multicolumn{2}{|c|}{$\begin{array}{c}\text { Weight* } \\
\text { (2) }\end{array}$}} & \multicolumn{2}{|c|}{ Output at factor cost } & \multirow{2}{*}{$\begin{array}{c}\text { Weight } \\
(5)\end{array}$} & \multicolumn{2}{|c|}{ Output at factor cost } \\
\hline & & & $\begin{array}{c}1954 / 55 \\
\text { (3) }\end{array}$ & $\begin{array}{l}1963 / 64 \\
(4)\end{array}$ & & $\begin{array}{l}1954 / 55 \\
\text { (6) }\end{array}$ & $\begin{array}{c}1963 / 64 \\
(7)\end{array}$ \\
\hline \multicolumn{2}{|c|}{ Non-metallic minerals } & 0 & - & - & 0.091 & 5,824 & 22,788 \\
\hline \multicolumn{2}{|c|}{ Basic metal industry } & 0 & - & - & 0.086 & 4,356 & 35,107 \\
\hline \multicolumn{2}{|l|}{ Metal } & 0 & - & - & 0.454 & 24,650 & 132,532 \\
\hline \multicolumn{2}{|c|}{ Electrical machinery } & 0 & - & - & 0.251 & 3,177 & 45,295 \\
\hline \multicolumn{2}{|l|}{ Transport } & 0 & - & - & 0.409 & 7,629 & 84,964 \\
\hline \multicolumn{2}{|c|}{ Miscellaneous } & 1 & 19,014 & 138,310 & 1.000 & 19,014 & 138,310 \\
\hline \multicolumn{2}{|l|}{ Total } & & $1,250,269$ & $4,271,907$ & & $1,390,158$ & $5,193,115$ \\
\hline \multicolumn{2}{|l|}{ Growth } & & \multicolumn{2}{|c|}{$242 \%$} & & \multicolumn{2}{|c|}{$274 \%$} \\
\hline
\end{tabular}

B. Intermediate Goods

\begin{tabular}{llrrrrr|} 
Edible oils & 0 & - & - & 0.187 & 17,237 & 107,393 \\
Cotton textiles & 0 & - & - & 0.127 & 80,230 & 255,311 \\
Jute textiles & 1 & 83,720 & 517,874 & 0.232 & 19,423 & 120,147 \\
Wood and furniture & 0 & - & - & 0.494 & 1,626 & 9,885 \\
Paper & 1 & 30,503 & 158,730 & 0.788 & 24,036 & 125,079 \\
Leather & 1 & 37,021 & 189,930 & 0.150 & 5,553 & 28,490 \\
Rubber & 1 & 13,273 & 42,265 & 0.255 & 3,385 & 10,778 \\
Fertilizer & 1 & 2,848 & 80,569 & 1.000 & 2,848 & 80,569 \\
Chemicals \& phar- & 1 & 36,280 & 261,670 & 0.280 & 10,158 & 73,268 \\
$\quad$ maceuticals & 1 & 71,951 & 223,010 & 0.170 & 12,232 & 37,912 \\
Petroleum & & - & - & 0.062 & 3,968 & 15,526 \\
Non-metallic minerals & 0 & - & - & 0.147 & 7,446 & 60,008 \\
Basic metal industry & 0 & - & - & 0.158 & 8,579 & 46,123 \\
Metal products & 0 & - & - & 0.028 & 354 & 5,053 \\
Electrical machinery & 0 & - & - & 0.053 & 989 & 11,010 \\
Transport & 0 & - & - & & 198,066 & 986,572
\end{tabular}


TABLE I (Contd.)

A WEIGHTED USE-CLASSIFICATION OF INDUSTRY OUTPUT

\begin{tabular}{|c|c|c|c|c|c|c|}
\hline \multirow{3}{*}{$\begin{array}{l}\text { Industry } \\
\text { (1) }\end{array}$} & \multicolumn{3}{|c|}{ Lewis and Soligo } & \multicolumn{3}{|c|}{ Our division } \\
\hline & \multirow{2}{*}{$\begin{array}{c}\text { Weight* } \\
\text { (2) }\end{array}$} & \multicolumn{2}{|c|}{ Output at factor cost } & \multirow{2}{*}{$\begin{array}{c}\text { Weight } \\
(5)\end{array}$} & \multicolumn{2}{|c|}{ Output at factor cost } \\
\hline & & $\begin{array}{c}1954 / 55 \\
\text { (3) }\end{array}$ & $\begin{array}{l}1963 / 64 \\
(4)\end{array}$ & & $\begin{array}{c}1954 / 55 \\
(6)\end{array}$ & $\begin{array}{c}1963 / 64 \\
(7)\end{array}$ \\
\hline \multicolumn{7}{|l|}{ C. Capital Goods } \\
\hline Wood and furniture & 0 & - & - & 0.228 & 750 & 4,562 \\
\hline Paper & 0 & - & - & 0.125 & 3,813 & 19,841 \\
\hline Non-metallic mineral & als 1 & 64,000 & 250,420 & 0.847 & 54,208 & 212,106 \\
\hline Basic metal industry & 1 & 50,656 & 408,220 & 0.767 & 38,853 & 313,105 \\
\hline Metal products & 1 & 54,297 & 291,920 & 0.388 & 21,067 & 113,265 \\
\hline $\begin{array}{l}\text { Machinery non- } \\
\text { electric. }\end{array}$ & 1 & 16,959 & 165,660 & 1.000 & 16,959 & 165,660 \\
\hline Electrical machinery & y 1 & 12,657 & 180,460 & 0.721 & 9,126 & 130,112 \\
\hline Transport & 1 & 18,653 & 207,737 & 0.538 & 10,035 & 111,763 \\
\hline Total & & 217,222 & $1,504,417$ & & 154,734 & $1,069,982$ \\
\hline Growth & & & $3 \%$ & & 59 & \\
\hline
\end{tabular}

* Weights were computed as proportion of total supply allocated to each category except: a) that where less than 2 per cent fell outside the principle category, the refinement was ignored and

b) that intermediate deliveries to construction were counted as deliveries to the capital goods category since Lewis and Soligo dealt only with manufacturing industry.

In addition to these two-the gross whole-industry classification used by Lewis and Soligo and the weighted classification we have used-a third alternative exists in the whole-industry classification used by Chenery [3]. This differs from Lewis and Soligo in that it classifies textiles as intermediate goods. Since Pakistan's cotton textile industry was, in 1954/55, larger by itself than all other consumption goods included in the Lewis and Soligo study and larger than all capital and intermediate goods combined, this is not an insignificant difference in classification.

In Table II the different descriptions of the composition of industrial structure which emerge from the three classifications are shown. It appears on $a$ priori grounds that treating cotton textiles as intermediate goods (Chenery) yields a rather suspicious structure of output. Both our classification system and that of Lewis and Soligo yield similar and seemingly more reasonable pictures of the 
structure of output - both in terms of composition and trend. There are, however, two differences worth noting: first, our classification yields a less radical shift in production structure during the $1954 / 55$ to $1963 / 64$ period; second, with regard to relative proportions, our classification indicates that the economy in $1963 / 64$ was in roughly the position which Lewis and Soligo argue it was starting from in $1954 / 55$.

Even if these differences were not significant, the basic virtue of this method would not be mitigated. The input-output based classification system has a better conceptual basis than those systems previously used. It allows us to call a spade a spade, and the results which it yields will, therefore, give a better picture of industrial structure.

TABLE II

THE STRUCTURE OF INDUSTRIAL OUTPUT

\begin{tabular}{ccccc}
\hline Sector & $1954 / 55$ & $1959 / 60$ & $1963 / 64$ \\
\hline
\end{tabular}

A: Lewis-Soligo Data, Our Classification

Consumption goods

$79.8 \%$

$76.0 \%$

$71.6 \%$

Intermediate goods

$11.4 \%$

$13.0 \%$

$13.4 \%$

Capital goods

$8.9 \%$

$11.0 \%$

$14.8 \%$

B: Lewis-Soligo Data, Lewis-Soligo Classification

Consumption goods

Intermediate goods

Capital goods
$71.7 \%$

$15.8 \%$

$12.5 \%$
$62.5 \%$

$21.6 \%$

$15.9 \%$
$58.9 \%$

$20.3 \%$

$20.7 \%$

\section{C: Lewis-Soligo Data, Chenery Classification}

Consumption goods

Intermediate goods

Capital goods
$33.5 \%$

$26.7 \%$

$29.7 \%$

$54.1 \%$

$57.4 \%$

$12.4 \%$

$15.9 \%$

$20.7 \%$ 


\section{REFERENCES}

1. Chenery, Hollis B., "Patterns of Industrial Growth", American Economic Review, Vol. L, No. 4, September 1960, pp. 624-654.

2. Lewis, Stephen R., Jr. and Ronald Soligo, "Growth and Structural Change in Pakistan's Manufacturing Industry, 1954 to 1964", Pakistan Development Review, Vol. V, No. 1, Spring 1965, pp. 95-139.

3. Pakistan, Planning Commmission, The Methodology of Estimating Import Requirements. (Karachi: Planning Commission, March 1963). 